AIP Applied Physics

\title{
Real time optical diagnostics of the plume dynamics during laser ablation of germanium in an oxygen environment
}

F. Vega, C. N. Afonso, and J. Solis

Citation: J. Appl. Phys. 73, 2472 (1993); doi: 10.1063/1.353106

View online: http://dx.doi.org/10.1063/1.353106

View Table of Contents: http://jap.aip.org/resource/1/JAPIAU/v73/i5

Published by the American Institute of Physics.

\section{Related Articles}

Negative effect of crystallization on the mechanism of laser damage in a $\mathrm{HfO} 2 / \mathrm{SiO} 2$ multilayer J. Appl. Phys. 112, 123103 (2012)

Anomalous transport of $\mathrm{Sb}$ in laser irradiated $\mathrm{Ge}$ Appl. Phys. Lett. 101, 172110 (2012)

Pulsed laser operated high rate charging of Fe-doped LiNbO3 crystal for electron emission J. Appl. Phys. 112, 073107 (2012)

Formation of nanostructured $\mathrm{TiO} 2$ by femtosecond laser irradiation of titanium in $\mathrm{O} 2$ J. Appl. Phys. 112, 063108 (2012)

Finite element calculations of the time dependent thermal fluxes in the laser-heated diamond anvil cell J. Appl. Phys. 111, 112617 (2012)

\section{Additional information on J. Appl. Phys.}

Journal Homepage: http://jap.aip.org/

Journal Information: http://jap.aip.org/about/about_the_journal

Top downloads: http://jap.aip.org/features/most_downloaded

Information for Authors: http://jap.aip.org/authors

\section{ADVERTISEMENT}

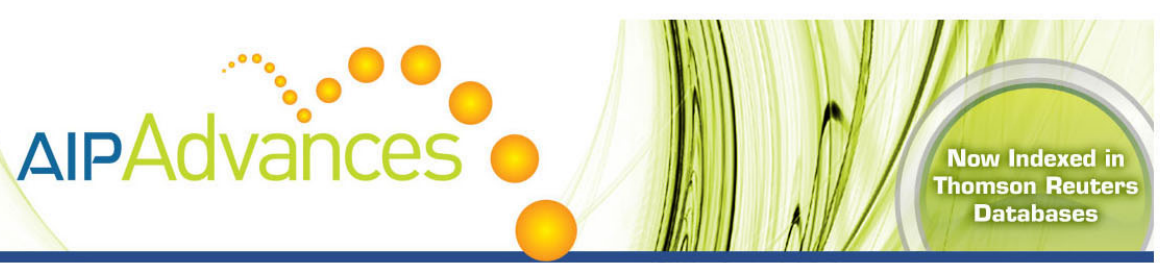

\section{Explore AIP's open access journal: Rapid publication \\ - Article-level metrics \\ Post-publication rating and commenting}




\title{
Real time optical diagnostics of the plume dynamics during laser ablation of germanium in an oxygen environment
}

\author{
F. Vega, C. N. Afonso, and J. Solis \\ Instituto de Optica (CSIC), Serrano 121, 28006 Madrid, Spain
}

(Received 27 May 1992; accepted for publication 9 November 1992)

\begin{abstract}
The dynamics of the gas phase induced by excimer laser ablation of Ge is investigated by analyzing the light emitted by the plume. Space and time-resolved optical spectroscopy measurements indicate the presence of both, neutral (GeI) and ionized (Gell) species. Two populations of neutrals with different velocities, which are related to the neutral atoms directly ejected from the target and those which are produced by recombination of ions, are observed. The velocities of the species remain unchanged for oxygen pressures up to $1 \mathrm{mbar}$, which suggest that the expansion of the plume occurs without further collisions with the foreign gas in this pressure range. The spectral emission characteristics are consistent with plume dynamics initiated by charged species.
\end{abstract}

\section{INTRODUCTION}

Laser ablation has been successfully applied to grow a great variety of materials in thin film configuration. ${ }^{1-3}$ Many efforts have been made to deposit complex oxide materials like high $T_{c}$ superconductors ${ }^{4,5}$ and ferroelectric films. ${ }^{6}$ The structure and composition of the laser deposited films depend on, among other experimental parameters, the laser fluence and wavelength ${ }^{7}$ and the background pressure. $^{8}$

It has been suggested that the whole deposition process is highly influenced by the dynamics of the species which are present in the plume formed by laser ablation of solid targets. Several diagnostic methods like time of flight, ${ }^{9-11}$ resonance ionization, ${ }^{12,13}$ optical emission ${ }^{14-16}$ laser induced fluorescence $e^{17,18}$ and resonant absorption ${ }^{19}$ have been used to study the ablation induced plume.

In ablation experiments using oxidized targets like $\mathrm{YBaCuO}$, oxide molecules in the plume were found which were either directly ejected from the target surface or formed by reactions in the gas phase. ${ }^{20}$ In addition, the nature and velocity of the species was very sensitive to the presence of a foreign gas. ${ }^{20-22}$ These features indicate the existence of collision processes in the plume which lead to the formation of oxide molecules and therefore seem to improve the deposition of stoichiometric films. ${ }^{23}$ In a separate work, ${ }^{24}$ we have shown that Ge oxide films can be grown by ablating a pure Ge target in an oxygen atmosphere. Since the presence of oxide compounds directly ejected from the target is ruled out in this case, the use of such a nonpreoxidated target may allow us to analyze whether the oxidation reaction occurs in the gas phase or at the substrate.

The aim of this work is to study the dynamics of the plume induced by laser ablation of a Ge target in an oxygen background pressure. We will analyze the light emitted by the plume in real time with space and spectral resolution in order to determine both the nature of the species and their velocity as they move ahead from the target surface. It will be shown that the plume is mainly initiated by charged species and that the velocity of both ions and neutrals does not depend on the oxygen pressure.

\section{EXPERIMENT}

The experimental setup is similar to that previously used for deposition of $\mathrm{GeO}_{x}$ thin films which is described in detail elsewhere. ${ }^{24,25}$ An ArF excimer laser [193 nm, 12 ns full width half maximum (FWHM)] is focused at normal incidence on a rotating Ge target $(99,999 \%)$. The laser repetition rate is $10 \mathrm{~Hz}$ and the energy at the target site is $0.03 \mathrm{~J}$ which yields to a fluence of $10 \mathrm{~J} / \mathrm{cm}^{2}$ when integrating the energy density profile at an intensity of 0.1 of its maximum value. ${ }^{25}$ The experiments are performed both in vacuum $\left(1.5 \times 10^{-5} \mathrm{mbar}\right)$ and in an oxygen pressure ranging from $10^{-4}$ to $10 \mathrm{mbar}$. This way we cover the pressure conditions which we have used previously to grow $\mathrm{GeO}_{x}$ thin films up to the stoichiometric value $(x=2){ }^{24}$

The light emitted by the plume is imaged onto the entrance slit of a SPEX spectrometer (wavelength resolution $0.05 \mathrm{~nm}$ ) by means of a lens and two mirrors resulting in a magnification of $\times 4$. One of the mirrors is set in a translation stage, allowing a well-controlled scan of the plume image across the entrance slit of the spectrometer. The final spatial resolution in the plume analysis is $160 \mu \mathrm{m}$. A photomultiplier ( $15 \mathrm{~ns}$ rise time) is connected to a boxcar averager (Stanford SR250) for spectrum recording or to a fast $7912 \mathrm{AD}$ Tektronix digitizer for transient emission measurements. The wavelength interval between 200 and $340 \mathrm{~nm}$ (where several emission bands of GeO molecules have been reported) ${ }^{26}$ was scanned, but no bands or lines which could be straightforwardly related to these molecules were found. In order to avoid second order contributions, we used glass optics and focused the present study to the $400-650 \mathrm{~nm}$ spectral range.

As we reported in Ref. 25, a diffraction effect at the edge of the target surface allows us to determine precisely the target surface position. This will be the spatial origin for the space resolved emission measurements. The time at 


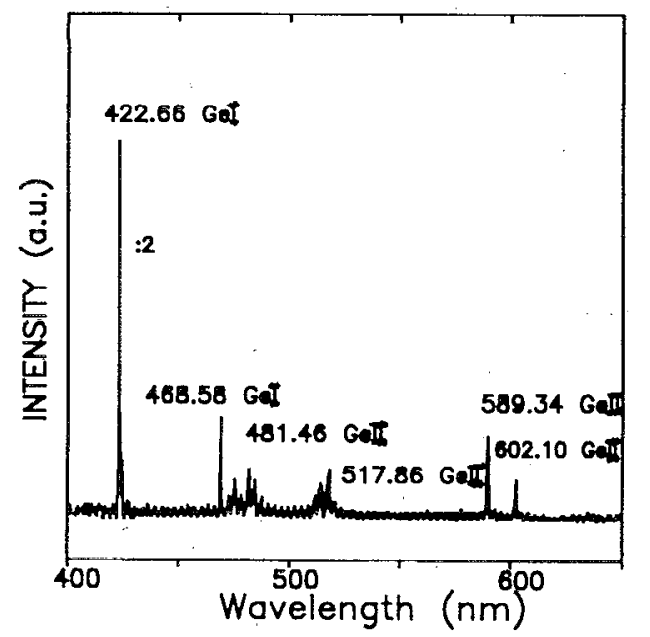

FIG. 1. Emission spectrum of the plume induced by laser ablation of a Ge target in vacuum. Some representative lines are labelled according to standard tabulation.

which the laser pulse reaches its maximum is taken as the temporal origin for the real time measurements.

\section{RESULTS}

The emission spectrum of the plume formed during laser ablation of a Ge target consists of several lines superimposed on an intense continuum. The continuum emission dissapears when moving $0.5 \mathrm{~mm}$ away from the target surface. At shorter distances its contribution to the spectrum is ruled out by delaying the boxcar gate around $75 \mathrm{~ns}$ after the maximum of the laser pulse. Figure 1 shows the emission spectrum of the Ge plume recorded in vacuum $\left(1.5 \times 10^{-5}\right.$ mbar) under these experimental conditions. The spectrum, recorded in an oxygen atmosphere up to 1 mbar, presents similar features. Most of the emission lines are identified according to standard tabulation ${ }^{27,28}$ and come from transitions involving neutral $\mathrm{Ge}(\mathrm{GeI})$ as well as single ionized Ge atoms (GeII). However, some of the lines could not be attributed to any known Ge transition. The existence of nontabulated lines in the recorded emission spectra during laser ablation of group IV elements has been explained by the presence of excited clusters. ${ }^{29}$ The nontabulated lines are not observed when the experiment is carried out at a lower laser energy density. Table I summarizes the lines identified in the emission spectra, the corresponding transitions, the energy of the upper level, and the lifetime of the excited state taken from Refs. 27, 28 and 30.

Each emission line is studied in real time at different distances from the target surface. As it has been already indicated, there is an intense continuum contribution to the transient emission at distances to the target surface shorter than $0.5 \mathrm{~mm}$. Figure 2 shows the time evolution of this continuum emission recorded out of any GeI or GeII lines together with the emission from $\mathrm{GeI}$ at $\lambda=422.66 \mathrm{~nm}$. The continuum emission intensity, which is stronger as the transient is recorded closer to the target surface, is formed
TABLE I. Emission lines which are observed in the Ge plume. The transitions involved, the energy of the upper transition level $\left(E_{k}\right)$ and the lifetime of the excited state are also included.

\begin{tabular}{lccrr}
\hline Spccic & $\begin{array}{c}\text { Wavelength } \\
(\mathrm{nm})\end{array}$ & Transition $^{\mathrm{a}, \mathrm{b}}$ & $\begin{array}{c}E_{k} \\
(\mathrm{cV})^{\mathrm{c}}\end{array}$ & $\begin{array}{c}\text { Lifetime } \\
(\mathrm{ns})^{\mathrm{b}}\end{array}$ \\
\hline GeI & 422.66 & $5 s^{1} P_{1}^{0}-4 p^{1} S_{0}$ & 4.96 & 47.6 \\
GeI & 468.58 & $5 s^{3} P_{1}^{0}-4 p^{1} S_{0}$ & 4.67 & 105.0 \\
GeII & 474.18 & $5 d^{2} D_{3 / 2}-5 p^{2} P_{1 / 2}^{0}$ & 12.41 & 21.7 \\
GeII & 481.46 & $5 d^{2} D_{5 / 2}-5 p^{2} P_{3 / 2}^{0}$ & 12.41 & 19.6 \\
GeII & 513.17 & $4 f^{2} F_{5 / 2}^{0}-4 d^{2} D_{3 / 2}$ & 12.44 & 5.0 \\
GeII & 517.86 & $4 f^{2} F_{7 / 2}^{0}-4 d^{2} D_{5 / 2}$ & 12.44 & 5.0 \\
& & $4 f^{2} F_{5 / 2}^{0}-4 d^{2} D_{5 / 2}$ & & \\
GeII & 589.34 & $5 p^{2} P_{3 / 2}^{0}-5 s^{2} S_{1 / 2}$ & 9.84 & 11.0 \\
GeII & 602.10 & $5 p^{2} P_{1 / 2}^{0}-5 s^{2} S_{1 / 2}$ & 9.79 & 12.0 \\
\hline \hline
\end{tabular}

${ }^{a}$ See Ref. 27

'See Ref. 30.

'See Ref. 28.

by an intense peak delayed respect to the laser pulse around 6-12 ns and a much less intense and broader one delayed around $600 \mathrm{~ns}$. The emission transient at $\lambda=422.66 \mathrm{~nm}$ shows clearly that two peaks are contributing to the emission. The less delayed one behaves similarly to that of the continuum emission at this wavelength. The longer delayed (around $100 \mathrm{ns)}$ ) and broader peak presents a long decay tail and corresponds to the emission due to the $5 s^{1} P_{1}^{0}-4 p^{1} S_{0}$ transition of GeI (see Table I).

The real time emission intensity for $\mathrm{GeI}$ at $\lambda=422.66$ $\mathrm{nm}$ recorded at different distances to the target surface is plotted in Fig. 3. It is clearly seen that the less delayed peak (6-12 ns) which was observed at short distances (see Fig. 2) disappears as the distance is increased $(>0.5 \mathrm{~mm})$. At these larger distances only the peak which corresponds to the transition of GeI above mentioned is observed. Its intensity broadens and its maximum occurs later as we move away from the target surface. The features observed both in the time profiles (Fig. 2) and their evolution as a function of the distance from the target (Fig. 3) give us

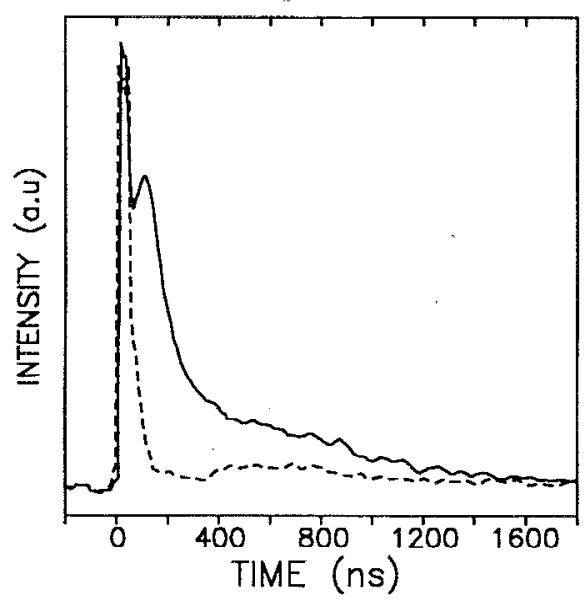

FIG. 2. Real time emission intensity recorded in vacuum and at $0.2 \mathrm{~mm}$ from the target surface: (dashed line) continuum emission recorded out of any identified line $(\lambda=424 \mathrm{~nm})$ and (solid line) emission recorded at a GeI line $(\lambda=422.66 \mathrm{~nm})$. 


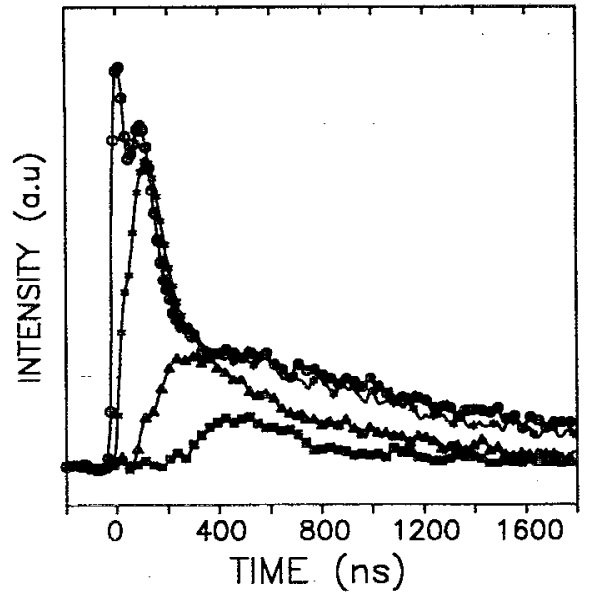

FIG. 3. Real time emission intensity recorded in vacuum at a Ger line $(\lambda=422.66 \mathrm{~nm})$ along the plume axis and at different distances to the target surface: (-O-O-O-) $0.2 \mathrm{~mm},\left(-*_{-} *_{-} *_{-}\right) 0.5 \mathrm{~mm},\left(-\Delta-\Delta_{-} \mathbf{A}_{-}\right) 1.5$ $\mathrm{mm}$, and (-1-in-) $3.0 \mathrm{~mm}$.

grounds to conclude that the less delayed peak observed in the emission at $\lambda=422.66 \mathrm{~nm}$ for short distances is caused by an overlapping of the continuum emission.

The features of the real time emission intensity transients for GeI taken at $\lambda=468.58 \mathrm{~nm}$ are qualitatively similar to those described above. For distances to the target surface shorter than $0.5 \mathrm{~mm}$, no emission at this wavelength was observed within the experimental sensitivity.

GeIr emission lines present common features which are different from those described for Gel lines. Figure 4 shows the temporal evolution of the emission intensity at $\lambda=589.34 \mathrm{~nm}$ at different distances from the target surface. At short distances, the emission consists of a sharp peak delayed respect to the laser pulse around $20 \mathrm{~ns}$ followed by a less intense, broader, and long delayed peak. The delay of this second weak peak is slightly dependent

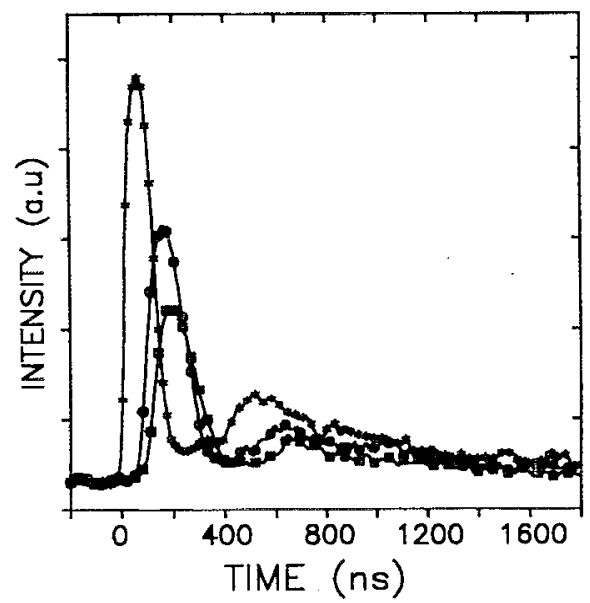

FIG. 4. Real time emission intensity recorded in vacuum at a GeIl line $(\lambda=589.34 \mathrm{~nm})$ along the plume axis and at different distances to the

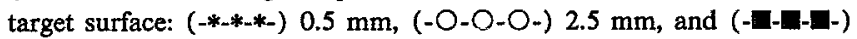
$3.0 \mathrm{~mm}$.

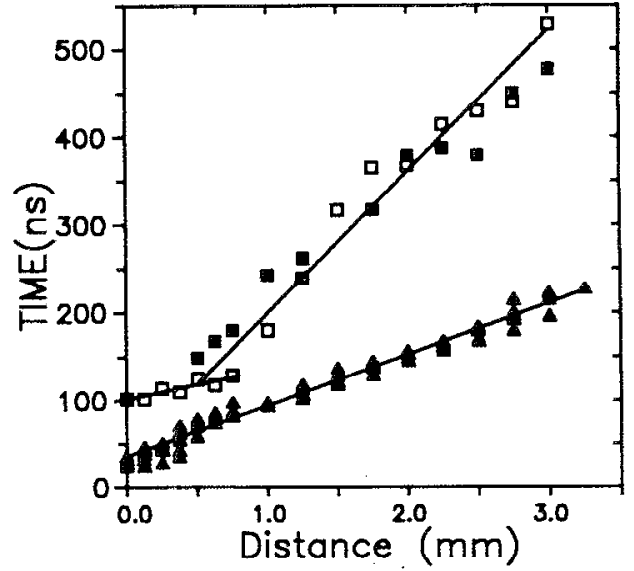

FIG. 5. Delay between the maximum emission intensity and the laser pulse as a function of the distance along the plume axis. It includes results for GeI ( $\square 22.66 \mathrm{~nm}, \square 468.58 \mathrm{~nm}$ ) and Gerr ( $A$ all identified lines in the 400-650 $\mathrm{nm}$ range).

on the particular Gell emission line monitorized. A contribution from the continuum may overlap the sharp peak for short distances but our temporal resolution does not allow us to separate it from the intense peak of GeII. Similarly to the GeI emission, the intensity maximum also occurs later and broadens as the emission is recorded along the plume axis and away from the target surface.

In Fig. 5, the delay between the laser pulse and the emission intensity maximum for both GeI and GeIr species is plotted versus the distance to the target surface. It is clearly scen that the ionized species are present in the plume before the neutral ones since their emission at the target surface is observed $20-30$ ns after the laser pulse while that from the neutrals appears $100 \mathrm{~ns}$ later. In addition the delay of the emission intensity maximum for ionized species presents a linear behavior as a function of the distance from the target at which the emission transient is recorded. For distances shorter than $0.75 \mathrm{~mm}$, a higher dispersion is observed in the experimental values, which may indicate the existence of either slower moving species or some kind of interactions among the ionized species. The inverse of the slope is the most probable velocity of the species flying along the target normal direction. This velocity, averaged among all the lines corresponding to GeII, is $1.7 \times 10^{6} \mathrm{~cm} / \mathrm{s}$, which is similar to the values usually reported for ionized species in other laser ablation experiments. ${ }^{31}$.

More complicated features are found in the velocity corresponding to the GeI species. At $\lambda=422.66 \mathrm{~nm}$ two velocity components are clearly seen. A fast component is predominant at distances to the target surface shorter than $0.75 \mathrm{~mm}$ corresponding to a velocity of $2.9 \times 10^{6} \mathrm{~cm} / \mathrm{s}$. At larger distances only a slow component is observed, which corresponds to a velocity of $6.2 \times 10^{5} \mathrm{~cm} / \mathrm{s}$. The latter value is again in the order of magnitude of those typically reported for neutral species in laser ablation experiments. ${ }^{32}$ The emission at $\lambda=468.58 \mathrm{~nm}$ exhibits only the slow component. 


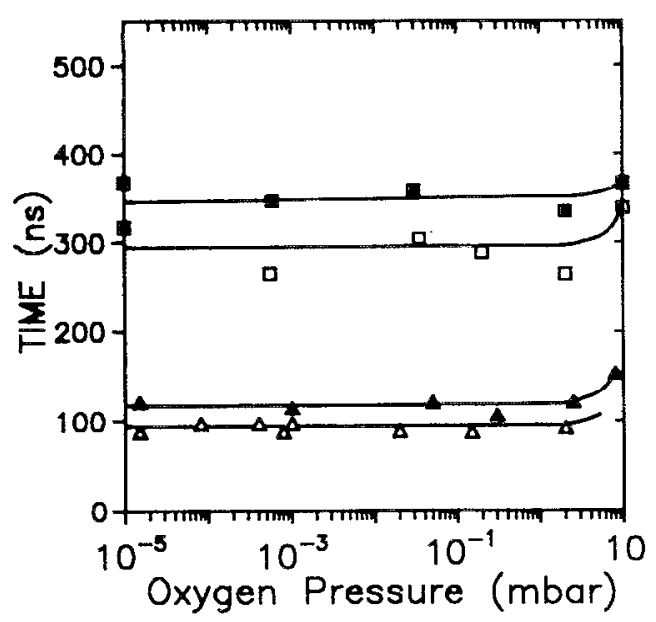

FIG. 6. Delay between the maximum emission intensity and the laser pulse as a function of the oxygen pressure and for different distances along the plume axis: $\square 1.5 \mathrm{~mm}, 2 \mathrm{~mm}$ for $\operatorname{GeI}(\lambda=422.66 \mathrm{~nm})$, and $\Delta 1.0$ $\mathrm{mm}, \Delta 2 \mathrm{~mm}$ for Gell $(\lambda=589.34 \mathrm{~nm})$.

The influence of the oxygen pressure in the plume dynamics is studied by measuring the delay of the emission intensity at different distances along the plume axis as a function of the oxygen pressure for each identified line. Figure 6 shows the results for GeI $(\lambda=422.66 \mathrm{~nm})$ and GeII $(\lambda=589.34 \mathrm{~nm})$ species. There is no change in the delay of the emission maximum when the oxygen pressure is increased up to $1 \mathrm{mbar}$. Although the results plotted in Fig. 6 correspond to only two of the emission lines, the same behavior is observed for all the lines found in the emission spectrum plotted in Fig. 1. According to what was discussed from Fig. 5, the velocity of the emitting species, both neutrals and ionized, remains constant in the presence of an oxygen environment in this wide oxygen pressure range.

\section{DISCUSSION}

The results are evidence that the species observed in the laser induced plume of $\mathrm{Ge}$ follow a complex dynamics. The observed emission features including the weak influence of the oxygen pressure, the faster velocity, and lower delay emission of the ionized species and the two velocity components in one of the neutral $\mathrm{Ge}$ emission lines, are the key to understanding the initial steps of the plume formation.

It has been reported that the irradiation of Ge with an ArF excimer laser induces a large emission of both electrons and ions from the target surface even at laser fluences just below the melting threshold. ${ }^{33}$ Our results are in agreement with the presence of charged species at the early stages of the plume formation. Taking into account the delay associated with the continuum emission (6-12 ns) and the laser pulse width $(\approx 12 \mathrm{~ns})$, it can be established that free electrons are able to be excited by absorbing some energy from the laser pulse itself via inverse bremsstrahlung. The intense continuum emission observed in the neighborhood of the target surface is then a consequence of free-free and free-bound electronic recombination. ${ }^{34}$ These excited electrons would induce the species emission through collisions. The earlier species in the Ge plume have to be mainly charged ones (electrons and ions) in agreement with the lower emission delay time measured for those species. This conclusion is further supported when the lifetime of the excited state (Table I) and the time at which the maximum intensity emission occurs at the target surface (Fig. 5) are compared. Both times are similar in the case of ions, whereas the former is shorter than the latter in the case of neutrals.

The evolution of the emission transients for GeI at $\lambda=422.66 \mathrm{~nm}$ as the emission is recorded ahead of the target surface shows the existence of two populations of neutrals which move at different velocities (Fig. 5). Several mechanisms for a two component velocity distribution have been proposed. The fast neutral species can be originated either by fast ions that recombine in the neighborhood of the target surface or by atoms directly ejected from the target. ${ }^{31}$ Since both processes are more likely at short distances, the fast species should dominate the emission near the target surface as we observe experimentally. The analysis of the delay of the emission intensity maximum suggests that the fast neutral population is more likely related to neutrals which are directly ejected from the surface, whereas the slow neutral population is produced from the recombination of ions.

For the emission at $\lambda=468.58 \mathrm{~nm}$ (GeI) the lifetime of the excited state ( $105 \mathrm{~ns}$ ) is of the same order of magnitude as the full width half maximum of the emission transients ( $120 \mathrm{~ns}$ at $0.5 \mathrm{~mm}$ ). Then GeI species excited to the $5 s^{3} P_{1}^{0}$ state are able to travel a measurable distance between excitation and emission, which may justify that no emission at $\lambda=468.58 \mathrm{~nm}$ could be recorded at distances from the target surface shorter than $0.5 \mathrm{~mm}$.

Finally, the velocity of the species in the plume remains constant up to 1 mbar oxygen pressure, which indicates that the interactions with the foreign atoms in the gas phase should be weak. Moreover there is no evidence of new emission lines when the spectrum is recorded in an oxygen environment which supports further that no new species (i.e., oxides) are formed in the plume. ${ }^{20}$ Only at oxygen pressures higher than $1 \mathrm{mbar}$, will the delay of the emission begin to increase, indicating that the dynamics of the plume become more complex. ${ }^{21,22}$ The laser-generated plasma has been modeled as a high pressure-temperature gas which expands anisotropically in vacuum. ${ }^{35}$ It has been shown that in the presence of a gaseous background for pressures typically below $1 \mathrm{mbar}$, the plume expansion is similar to a free expansion in vacuum since the mass of the ablated products is large compared to that of the driven gas. ${ }^{22,36,37}$ This condition applies in our case and can account for the nondependence of the species velocity on the oxygen pressure and therefore the absence of reactions in the gas phase. At higher background pressures, shock waves $^{38}$ are produced and the plume expansion becomes affected by the gaseous background ${ }^{36,39}$ according to our experimental results (see Fig. 6). A comparison of the present results with those reported elsewhere, ${ }^{24}$ which 
shows that stoichiometric $\mathrm{GeO}_{2}$ films are grown for oxygen pressures higher than $10^{-3} \mathrm{mbar}$, evidence that the incorporation of oxygen in the Ge oxide films is mainly due to reactions at the substrate.

\section{CONCLUSIONS}

The plume induced by irradiating a Ge target in an oxygen environment with an ArF excimer laser is initiated by the emission of charged species (electrons and ions). Two populations of neutrals with different velocities are observed. The fast species, both neutral and ionized, are produced by direct ejection from the target whereas the slower species (neutrals) could be originated by recombination of ions. The velocity of the species is determined by the initial expansion conditions and remains constant under the presence of an oxygen pressure $(<1 \mathrm{mbar})$. The expansion of the plume is collisionless with the foreign oxygen atoms or molecules in this pressure regime.

\section{ACKNOWLEDGMENTS}

This work was partially supported by CICYT (Spain) under the TIC-90 programme. We want to thank C. de Francisco and M. A. Garcia (Instituto de Optica, CSIC) for technical assistance.

${ }^{1}$ H. Tabata, T. Kaway, S. Kaway, O. Murata, J. Fujioka, and S. Minakata, Appl. Phys. Lett. 59, 2354 (1991).

${ }^{2}$ K. E. Youden, R. W. Eason, M. C. Gower, and N. A. Vainos, Appl. Phys. Lett. 59, 1929 (1991).

${ }^{3}$ S. Ferrer, F. Comin, J. A. Martin, L. Vazquez, and P. Bernard, Surf. Sci. 251/252, 960 (1991).

${ }^{4}$ D. Dijkamp, T. Venkatesan, X. D. Wu, S. A. Shaheen, N. Jisrawi, Y. H. Min-Lee, W. L. Mclean, and M. Croft, Appl. Phys. Lett. 51, 619 (1987).

${ }^{5}$ W. Ludfor, X.-Z. Wang, and D. Bäuerle, Appl. Phys. A 49, 221 (1989).

${ }^{6}$ S. Otsubo, T. Maeda, T. Minamikawa, Y. Yonewaza, A. Morimoto, and

T. Shimizu, Jpn. J. Appl. Phys. 29, 133 (1990).

${ }^{7}$ K. Ramkumar, J. Lee, A. Safari, and S. C. Danforth, Mater. Res. Soc. Sym. Proc. 200, 121 (1990).

${ }^{8}$ E. Namuri, J. Lee, C. Li, S. Hosokawa, S. Patel, and D. T. Shaw, Appl. Phys. Lett. 59, 3180 (1991).

${ }^{9}$ H. Izumi, K. Ohata, T. Sawada, T. Morishita, and S. Tanaka, Appl. Phys. Lett. 59, 597 (1991).

${ }^{10}$ G. Wedlwer and H. Ruhmann, Surt. Sci. 121, 464 (1982).

${ }^{11}$ H. Helvajian and R. Welle, J. Chem. Phys. 91, 2616 (1989).
${ }^{12}$ S. G. Hansen, J. Appl. Phys. 66, 1411 (1989).

${ }^{13}$ U. Krönet, St. Becker, Th. Hilberath, H.-J. Kluge, and C. Schulz, Appl. Phys. A 44, 339 (1987).

${ }^{14}$ X. Chen, J. Mazumder, and A. Purohit, Appl. Phys. A 52, 328 (1991).

${ }^{15}$ O. Auciello, S. Athavale, O. E. Hankins, M. Sito, A. F. Schreiner, and N. Biunno, Appl. Phys. Lett. 53, 72 (1988).

${ }^{16}$ J. P. Zheng, Q. Huang, T. Shaw, and H. S. Kwok, Appl. Phys. Lett. 54, 280 (1989).

${ }^{17}$ R. W. Dreyfus, J. Appl. Phys. 63, 1721 (1991).

${ }^{18}$ C. E. Otis and R. W. Dreyfus, Phys. Rev. Lett. 67, 2102 (1991).

${ }^{19}$ N. H. Cheung, Q. Y. Ying, J. P. Zheng, and H. S. Kowk, J. Appl. Phys. 69, 6349 (1991).

${ }^{20}$ D. Fried, G. P. Reck, T. Kushida, and E. W. Rothe, J. Appl. Phys. 70, 2337 (1991).

${ }^{21}$ C. Girault, D. Damiani, J. Aubreton, and A. Catherinot, Appl. Phys. Lett. 54, 2035 (1989).

${ }^{22}$ P. E. Dyer, A. Issa, and P. H. Key, Appl. Surf. Sci, 46, 89 (1990).

${ }^{23}$ T. Venkatesan, X. D. Wu, A. Inam, Y. Jeon, M. Croft, E. W. Chase, C. C. Chang, J. B. Wachtman, R. W. Odom, F. Radicati, and C. A. Magee, Appl. Phys. Lett. 53, 1431 (1988).

${ }^{24}$ F. Vega, J. C. Sande, C. N. Afonso, C. Ortega, and J. Siejka, Appl. Opt. (to be published).

${ }^{25}$ C. N. Afonso, F. Vega, J. Solis, F Catalina, C. Ortega, and J. Siejka, Appl. Surf. Sci. 54, 175 (1992).

${ }^{26} \mathrm{R}$. W. B. Pearse and A. G. Gaydon, The Identification of Molecular Spectra (Chapman and Hall, London, 1976).

${ }^{27}$ K. L. Andrew and K. W. Meissner, J. Opt. Soc. Am. 49, 146 (1959).

${ }^{28}$ J. Reader, C. Corliss, W. L. Wiese, and G. A. Martin, Eds., Wavelengths and Transitions Probabilities for Atoms and Atomic Ions (Nat. Stand. Ref. Data Ser., Nat. Bur. Stand., Washington DC, 1980), Vols. 1 and 2.

${ }^{29}$ A. Kasuya and Y. Nishina, Mater. Res. Soc. Symp. Proc. 191, 73 (1990).

${ }^{30}$ C. E. Moore, Atomic Energy Levels As Derived From the Analyses of Optical Spectra (Nat. Stand. Ref. Data Ser., Nat. Bur. Stand., Washington DC, 1971), Vol. 2.

${ }^{31}$ K. L. Saenger, J. Appl. Phys. 66, 4435 (1989).

${ }^{32}$ H. Wang, A. P. Salzberg, and B. R. Weiner, Appl. Phys. Lett. 59, 935 (1991).

${ }^{33}$ M. M. Bialkowski, G. S. Hurst, J. E. Parks, D. H. Lowndes, and G. E. Jellison, Jr., J. Appl. Phys. 68, 4795 (1990).

${ }^{34}$ H. R. Griem, Mater. Sci. Eng. A 139, 1 (1991).

${ }^{35}$ R. K. Singh and J. Narayan, Phys. Rev. B 41, 8843 (1990).

${ }^{36}$ A. Gupta, B. Braren, K. G. Casey, B. H. Hussey, and R. Kelly, Appl. Phys. Lett. 59, 1302 (1991).

${ }^{37}$ W. Marine, M. Gerry, J. M. Scotto d'Aniello, M. Sentis, P. Delaporte, B. Forestier, and B. Fontaine, Appl. Surf. Sci. 54, 264 (1992).

${ }^{38}$ Y. A. Zeldovitch and Y. P. Raizer, Physics of Shock Waves and High Temperature Hydrodynamics Phenomena (Academic, New York, 1967), Vol. 1.

${ }^{39}$ P. E. Dyer, A. Issa, and P. H. Key, Appl. Phys. Lett. 57, 186 (1990). 